\title{
Reflets
}

Revue ontaroise d'intervention sociale et communautaire

\section{Entrevue avec Alan Moscovitch}

\section{Adje van de Sande et Nérée St-Amand}

Volume 7, numéro 2, automne 2001

Le travail social en Ontario

URI : https://id.erudit.org/iderudit/026354ar

DOI : https://doi.org/10.7202/026354ar

Aller au sommaire du numéro

Éditeur(s)

Reflets : Revue ontaroise d'intervention sociale et communautaire

ISSN

1203-4576 (imprimé)

1712-8498 (numérique)

Découvrir la revue

Citer ce document

van de Sande, A. \& St-Amand, N. (2001). Entrevue avec Alan Moscovitch. Reflets, 7(2), 16-27. https://doi.org/10.7202/026354ar

Tous droits réservés (C) Reflets : Revue ontaroise d'intervention sociale et communautaire, 2001
Ce document est protégé par la loi sur le droit d'auteur. L'utilisation des services d’Érudit (y compris la reproduction) est assujettie à sa politique d'utilisation que vous pouvez consulter en ligne.

https://apropos.erudit.org/fr/usagers/politique-dutilisation/ 


\section{Entrevue avec Alan Moscovitch}

Cette entrevue a été réalisée par Adje van de Sande et Nérée St-Amand. Ils ont rencontré Alan Moscovitch, le 25 septembre 2001. Reflets tient à remercie Alan Moscovitch d'avoir accepté de faire cette entrevue et de partager ses opinions et ses analyses dans le cadre de ce numéro qui traite de l'intervention sociale en Ontario français. Grâce à son expérience dans le domaine social depuis plus de 25 ans, nous nous proposons de brosser un portrait de la profession avec ses défis, ses priorités, ses marges de manouvre et ses contraintes, dans un univers de plus en plus complexe et tiraillé entre des valeurs et des pratiques qui remettent en question nos formations et nos croyances.

Reflets: Commençons, si vous le voulez bien, par quelques questions historiques, avant d'arriver aux défis actuels de notre profession. D'après vous, quels sont les événements sociaux des trente dernières années ayant le plus transformé la profession du service social?

Moscovitch: Un événement a marqué de façon significative le domaine social et la profession du service social, au début des années soixante. Il s'agit de l'adoption, en 1966, du Régime d'assistance publique du Canada (RAPC). En adoptant cette loi, le gouvernement fédéral a joué un rôle décisif dans le domaine social et transformé ses rapports avec les provinces. En effet, pour la première fois de notre histoire, le gouvernement fédéral a décidé d'assumer, en donnant pratiquement carte blanche aux provinces, la moitié des dépenses dans le domaine social. Il disait ainsi aux provinces et à la population canadienne son engagement à défrayer la moitié des sommes encourues dans les domaines de l'éducation, de la santé et des services sociaux. Une telle initiative se situait dans une conjoncture bien particulière:la montée de l'État providence et son engagement dans la lutte à la pauvreté. Durant la période d'âge d'or du développement social qui a suivi, le gouvernement fédéral a financé à part égale de nombreux programmes pour 
prévenir la pauvreté, améliorer la santé, protéger les populations défavorisées et diverses couches sociales de la société canadienne. Mais, on doit aussi se rappeler que l'accord précisait que les provinces devaient d'abord s'engager à défrayer les coûts des programmes et en réclamer après la moitié au fédéral.

Suite à cet accord, à la fin des années soixante et au début des années soixante-dix en particulier, les provinces ont donc augmenté de façon significative les dépenses dans le domaine social. Ceci a entrainé la formation et l'embauche d'un nombre impressionnant de personnes pour gérer ces programmes (assistance sociale, enfance, jeunesse, projets communautaires, santé, santé mentale, etc.). Le service social s'est alors affirmé comme une profession d'aide, mandatée pour s'occuper d'individus, de groupes et de communautés visées par ces programmes d'assistance. Nous avons alors vu, jusque dans les années 1980, une augmentation très importante du nombre de personnes travaillant dans les services sociaux et du nombre de programmes institués par les provinces et territoires pour réduire les inégalités sociales.

Cette loi est demeurée en vigueur pendant trente ans, soit de 1966 à 1996. Au cours de cette période, des fonds importants ont été consacrés à l'augmentation des services, des programmes, des projets sociaux dans l'ensemble des provinces et territoires.

Reflets: Selon vous, quel bilan pouvons-nous faire des actions des gouvernements durant cette période? Pouvez-vous partager avec nous votre analyse de l'intervention de l'État providence dans le domaine du social?

Moscovitch: Après 1966, nous avons vu une augmentation des dépenses gouvernementales dans le champ du bien-être en général, que ce soit la santé, l'éducation en incluant le niveau collégial et universitaire, mais aussi dans des programmes de soutien comme le bien-être social et les garderies de jour. En 1973, il y a eu un changement dans la réglementation de la loi. Pourtant, il ne faut pas oublier qu'ont est dans une période d'expansion et que les provinces cherchaient, de plus en plus, à rendre conforme leurs services à la loi sur le Régime d'assistance publique pour bénéficier du partage des coûts avec le gouvernement fédéral. Et l'expansion 
des services s'est poursuivie jusqu'au début des années 1980, principalement durant la période où le Parti Libéral était au pouvoir, à Ottawa.

Sous le gouvernement fédéral conservateur, nous avons assisté aux premières tentatives de réduction des dépenses, de compressions budgétaires. En 1984, le gouvernement Mulroney avait annoncé son plan de réduction des dépenses, spécialement au chapitre des programmes de bien-être social. Ce changement d'orientation était basé sur les résultats de la Commission royale d'enquête sur l'économie (Rapport Macdonald), créée par le gouvernement libéral antérieur. Or, un fait ressort, les Conservateurs ont eu beaucoup de difficultés à effectuer ces changements. Ils ont rencontré énormément de résistance pendant toute la période s'étendant de 1984 à 1993, année où ils ont été défaits. Cette défaite s'inscrit justement dans le contexte d'une campagne nationale visant à réduire le déficit et avec l'objectif de s'attaquer aux dépenses effectuées dans le domaine du social. Chose curieuse, le gouvernement libéral qui est revenu au pouvoir en 1993, a réussi à implanter les réformes proposées par le gouvernement conservateur précédent.

Il faut dire que ces réformes qui n'avaient pas été possibles, avaient rencontré des résistances de taille, en particulier au niveau de la loi sur l'assurance-chômage. Progressivement, depuis 1993, dans la foulée de la remise en question de l'État providence, on assiste au démantèlement du RAPC. Les changements dans les programmes d'assistance sociale ont fait beaucoup de bruit. Par exemple, en Ontario, n'oublions pas les coupures dans le système de santé, l'éducation et le logement social, en particulier.

En 1996, le gouvernement libéral a adopté une législation leur permettant de mettre fin au RAPC. Il a alors effectué des réductions importantes dans tous les domaines. La santé, l'éducation et le service social ont été la scène de coupures budgétaires drastiques.

Reflets: Mais pourquoi le gouvernement conservateur a-t-il été incapable d'effectuer ces coupures, alors que le gouvernement libéral a réussi à les implanter? 
Moscovitch: C'est une question intéressante. Après la Seconde Guerre Mondiale, on semblait accepter l'idée que l'État providence et ses programmes d'assurance contre certaines éventualités (chômage, accidents, problèmes de santé, problèmes familiaux) étaient bénéfiques pour l'économie, grâce à l'économiste Keynes, en particulier. Durant les années soixante, les gens ont donc accepté d'emblée l'idée de l'État providence, qui nécessitait un gouvernement central fort ainsi qu'une intervention gouvernementale majeure dans l'économie par le biais de différents programmes. Ces derniers ont toutefois nécessité une période de temps pour être implantés. En effet, l'État a mis de cinq à dix ans simplement pour mettre en place l'infrastructure, les institutions nécessaires pour gérer les programmes comme le système national de santé.

Si nous parlons de la période du gouvernement conservateur, nous parlons de quelques années après ces grands changements. Au début, des résistances très importantes se sont fait sentir.Je me rappelle très bien, en 1985 par exemple, lors d'une manifestation sur la colline parlementaire contre les changements dans le régime de pension de retraite, un journaliste de Radio-Canada avait demandé à une femme pourquoi elle participait à cette démonstration. Elle avait expliqué qu'elle voulait conserver sa «pension» du fédéral. Pour elle, c'était important. Ses paroles ont-elles porté fruit? "If the government doesn't understand that, then its goodbye Charlie Brown». La semaine suivante, le gouvernement conservateur a annoncé qu'il suspendait les changements prévus au régime de pension du Canada. Il a compris assez vite le message qu'un gouvernement qui veut rester au pouvoir, ne doit pas toucher aux programmes comme les pensions de retraite parce qu'il y a beaucoup de gens qui en dépendent. Donc, c'était une décision bien plus politique qu'idéologique.

Reflets: Si vous le voulez bien, passons à la seconde partie de notre entretien, soit l'état de la profession et l'avenir du service social?

Moscovitch: À mon avis, l'état de la profession est étroitement lié aux dépenses du gouvernement dans le domaine social. En effet, au Canada, 
quand on parle de service social, on parle de services publics; le service social privé représente un petit nombre d'emplois en comparaison avec les gens embauchés dans les fonctions publiques. Même avec les coupures budgétaires en travail social, il reste encore beaucoup de gens qui sont actuellement employés dans les services publics.

Est-ce que la profession est en bonne santé? Difficile à dire. Au cours des quinze dernières années, on a assisté à plusieurs changements qui ont affecté la disponibilité des services, mais si on regarde en gros, on constate que la plupart des agences et des organismes gérés par les gouvernements provinciaux continuent à embaucher un très grand nombre de gens dans la pratique, dans le champ comme on dit.

Quel est le défi pour la profession? Je le situerais en parallèle avec les autres professions reconnues, celles qui ont passé à travers les défis que nous connaissons actuellement. Certaines professions possèdent des critères rigoureux d'entrée dans la pratique, d'admission à la profession. Par exemple, le Collège des médecins détermine les conditions d'admission au collège. Ce sont des médecins qui décident qui sera médecin, que ce soit en pratique privée, dans les hôpitaux ou les cliniques communautaires. Ces postes sont réservés à ceux et celles qui peuvent démontrer qu'ils sont médecins selon le collège. Tel n'est pas le cas en travail social. Nous ne sommes pas en mesure de dire: «Moi, je suis travailleuse sociale». Nous sommes plus proches de cette situation dans certaines provinces, mais nous sommes loin de déterminer qui peut remplir certains postes dans le champ, dans certains lieux de pratique. On est loin de ça. Par exemple, il est encore permis qu'une agence de l'Aide à l'enfance embauche une personne qui n'a pas les qualifications de travailleur social et de lui donner leur propre programme de formation. Ils ne sont obligés en rien. Ils peuvent choisir parmi les gens qui possèdent diverses qualifications au niveau collégial, au niveau d'un baccalauréat ou même d'une maitrise. En fait, nous faisons face à deux problèmes: premièrement, les postes à combler restent très ouverts pour les employeurs qui ont énormément de pouvoir discrétionnaire et, deuxièmement, c'est un problème pour notre profession auquel elle ne veut pas 
faire face. Pour nous, le défi consiste à définir le type de formation requis pour un travail, et quel genre de pratique convient à une formation collégiale, un baccalauréat ou une maitrise. Et la profession n'a pas encore défini exactement les paramètres qui s'appliquent à l'un ou à l'autre. Est-ce que c'est nécessaire, par exemple, pour certaines interventions dans le domaine de l'aide à l'enfance d'avoir une maitrise? Est-il acceptable dans d'autres domaines d'accompagnement d'exiger des qualifications collégiales ou une éducation postsecondaire? La profession du service social ne peut pas encore le déterminer. On ne sait pas parce qu'on n'a pas fait la distinction entre la formation collégiale et les formations universitaires de baccalauréat ou de maitrise.

Reflets: D'après ce que vous dites, il devrait y avoir plus de critères précis. Mais, à ce moment là, où est la place de la réflexion, de l'initiative personnelle, si chacun définit des actes selon les critères des agences?

Moscovitch: D'accord, mais pour l'instant, la profession a laissé tout ça ouvert. Et la conséquence, c'est que tout un chacun peut faire à peu près n'importe quoi. Ceci n'est pas la seule raison, certes, mais, parce que c'est ouvert, on peut choisir entre diverses professions et diverses formations pour accomplir des tâches bien spécifiques. Parce que nous ne sommes pas en mesure de définir les différentes tâches liées à chaque niveau de formation, nous laissons le soin à d'autres individus ou à d'autres professions de faire un travail qui correspond à nos spécialisations.

Reflets: Il y a des tâches, mais il y a aussi des problèmes sociaux complexes. Par exemple, il est très difficile de définir chacune des tâches lorsqu'on parle d'abus contre les enfants et de la violence familiale ou conjugale. Comment une seule formation ou un seul niveau de formation peut-il aborder cette complexité et voir les choses dans leur ensemble, en tenant compte des dimensions sociales, politiques, économiques, politiques d'un problème?

Moscovitch: L'employeur a aussi une tâche à accomplir. Je dis simplement que c'est un défi pour la profession de définir les particularités des différentes qualifications, sinon, nous restons là où nous sommes. Sans définition de nos spécialisations, nous demeurons très 
vulnérables. Par exemple, est-ce important d'avoir quelqu'un avec une maîtrise et un certain nombre d'années d'expérience pour travailler avec certains cas de violence ou est-il possible qu'une personne possédant d'autres qualifications que les nôtres puisse le faire? Dans le cas des autres professions, ils insistent sur le fait qu'il faut avoir des qualifications précises pour effectuer une tâche.

Reflets: Mais faut-il se guider sur l'exemple des autres professions? La psychologie, la médecine sont-elles nécessairement nos modèles?

Moscovitch: Je dis simplement que si la profession n'aborde pas la question, elle va rester ouverte. Si elle ne reçoit pas de réponse, il y aura un vide qui sera comblé par les employeurs. Ils seront obligés de combler les postes et ils le feront à leur façon. À mon avis, il faut prendre des décisions sur les qualifications et l'expérience requises pour intervenir dans une situation.

Reflets: Ceci nous amène à vous poser la question de votre vision du travail social. Est-ce qu'elle correspond aux attentes imposées par les milieux de travail tels que les Sociétés d'aide à l'enfance, les Centres d'accès aux soins communautaires ou encore les services de probation?

Moscovitch: Pas tout à fait. J'ai une vision différente des moyens de livraison de services. Ce qui manque, je crois, c'est un système national de normes. Comment peut-on arriver avec ce genre de normes? Il y avait un système de normes dans le Régime d'assistance publique et à mon avis, ce fut l'un des avantages du régime durant la période de 1966 à 1996. Maintenant, on manque de normes. Mais est-ce bien nécessaire d'avoir une autre loi fédérale, dans le contexte politique actuel? De toute façon, ce serait probablement impossible. Par contre, il serait possible d'avoir un accord entre les provinces et le gouvernement fédéral sur des normes. Et c'est le but des discussions actuelles sur le contrat social. Au niveau provincial, je préférerais voir un système de livraison standard, c'est-à-dire un système dans lequel on a des normes de service pour la population et on assure que les services seront disponibles à travers les centres de service social, chargés de la livraison dans chaque province. Évidemment, les services seront différents dans chaque province, 
mais en général, ce que je vois, c'est un système où on peut garantir la livraison d'un ensemble de service, d'un ensemble clef de service. Je concède qu'il devrait y avoir des variations dans chaque région, territoire ou province. Les citoyens s'adaptent et ils vont adapter les différentes agences ou organisations pour qu'elles rendent disponibles les services importants dans chaque région. Ce que je veux voir, c'est un ensemble clef de services offerts dans chacune des provinces.

Reflets: D'après ce que nos étudiants et étudiantes nous disent, il existe un écart entre ce qu'ils apprennent dans la salle de classe et ce qu'ils apprennent dans leur milieu de stage. En d'autres mots, ils accusent la formation académique de ne pas être au diapason des préoccupations de la pratique. Que pensez-vous de telles préoccupations?

Moscovitch: Question souvent posée, n'est-ce pas? Pour moi, je soutiens que le rôle de l'université ne consiste pas simplement à préparer des gens pour le marché du travail. Ce n'est pas ce genre de formation que je privilégie. Je ne nie pas l'importance de ce genre de formation, mais je crois que ce n'est pas le rôle des programmes universitaires de préparer les gens directement pour tel ou tel travail ou de donner toutes les connaissances nécessaires pour oeuvrer dans tel ou tel champ. De toute façon, c'est impossible de le faire à l'université. Celle-ci ne peut pas préparer les étudiants pour tous les différents emplois possibles. À mon avis, le rôle de l'université est de préparer des gens qui peuvent penser et ensuite, solutionner des problèmes. Ça, c'est le rôle de l'université. Le rôle de l'agence et de l'institution, avec ses préoccupations centrées sur le service, consiste à ajouter les connaissances spécifiques pour travailler dans telle ou telle agence, dans tel volet de la profession.

Reflets: Parfois, on pourrait croire qu'il y a harmonie ou complémentarité entre les deux genres d'apprentissage. Mais ce n'est pas toujours le cas, n'est-ce pas?

Moscovitch: Nos sociétés ont pour fondement la libre expression. Et les universités sont, en principe, basées sur le même principe.Ainsi, il se peut fort bien qu'il y ait des différences dans les façons 
d'entrevoir les problèmes, selon qu'on favorise une approche plus académique ou plus professionnelle. À mon avis, ces différences sont importantes, essentielles, car elles servent à aiguiser la pensée des étudiants, des travailleurs et même, des universitaires. C'est possible, même pour nous, universitaires, d'apprendre à travers de telles démarches.

Reflets: Mais il semble que les tensions soient devenues plus grandes, étant donné que les employeurs semblent vouloir dicter davantage le genre d'apprentissage qu'ils attendent à la sortie des études. Par ailleurs, les gouvernements aussi restreignent jusqu'à un certain point les formes d'apprentissage au plan idéologique. Nous sentons des pressions allant vers la performance. La profession n'est-elle pas prise en souricière entre ces deux contraintes?

Moscovitch: Je crois que ces éléments ont toujours été là. Durant les derniers cinquante ans, et même avant, lors de la Grande Dépression de 1930 par exemple, il y avait des écoles de pensée au sein de la profession qui prétendaient qu'il était important de travailler tout simplement avec la famille ou le couple et de trouver des solutions à leurs problèmes de communication au lieu de proposer des approches qui analysaient la pauvreté de la personne touchée par le chômage. L'histoire nous révèle qu'il y avait alors des divergences d'idées, des débats de fond au sein de notre profession et ils subsistent encore, c'est certain. Ils ne sont pas nouveaux. La profession est tiraillée entre différentes façons de penser, différentes idéologies, différentes écoles qui interprètent différemment les problèmes des gens.

Dans un tel contexte, il est essentiel, à mon avis, pour les universités de garder leur indépendance, de choisir les apprentissages qu'elles considèrent importants et d'insister sur les connaissances universitaires, sans pour autant négliger les habiletés nécessaires à la pratique. Le tout doit se faire avec une certaine distance vis-à-vis les demandes des institutions, des agences et les préoccupations qu'on pourrait qualifier d'institutionnelles. 
Reflets: Avec un gouvernement conservateur comme celui que nous avons actuellement, les politiques néo-libérales ont préséance, n'est-ce pas? Ceci met-il davantage de pressions pour ajuster la formation aux contraintes des organismes subventionnaires et aux visées gouvernementales?

Moscovitch: Nous subissons des pressions importantes actuellement parce que le gouvernement ontarien a décidé de poursuivre une avenue qui s'oppose aux valeurs de justice et d'équité de notre profession. Il y a eu certes des changements importants. Par exemple, il a effectué des coupures importantes dans les budgets des Sociétés d'aide à l'enfance, mais il a aussi réalisé, par la suite, que les Sociétés ne pouvaient pas fonctionner avec si peu de personnel, que des tragédies pouvaient survenir et des vies humaines en souffrir.Ainsi, nous avons été témoins de pressions, pas seulement de la part des institutions et des différentes agences d'aide à l'enfance, mais aussi de la part du public. Au fond, les gouvernants veulent simplement éviter les problèmes, mais ils ne veulent pas être perçus comme des dirigeants qui ne sont pas préoccupés par les problèmes des gens, en particulier ceux des enfants. Ils veulent éviter que le public les perçoive comme des gens insensibles, mais ils veulent aussi que les problèmes disparaissent: «They want to make the problem go away". Donc, même un gouvernement avec une approche idéologique de droite est obligé de faire face à la réalité et s'occuper des problèmes.

Reflets: Parce que le gouvernement réalise qu'il $y$ a des conséquences, j'imagine. Bon, si nous résumions la situation de la profession maintenant, quelle est la situation de la profession du service social, surtout en Ontario?

Moscovitch: Présentement, la plupart des gens sont obligés de travailler dans des institutions, pour des services gouvernementaux.Actuellement, les gouvernements n'ont pas la réputation d'adopter une approche idéologique qui colle bien aux valeurs de la profession. D'une certaine façon, la profession attend un changement au niveau politique. Elle attend de savoir si d'autres argents, d'autres fonds seront disponibles pour augmenter certains services. On espère avoir un prochain gouvernement qui saura mettre de l'avant les besoins de la citoyenneté. 
Reflets: Qu'est-ce qui s'annonce pour l'avenir de notre profession?

Moscovitch: C'est difficile à dire. En raison des changements démographiques, on peut prédire qu'il y aura certains changements au niveau de la demande pour les services. Par exemple, notre population devient de plus en plus âgée. Il y aura donc une demande accrue pour des travailleuses spécialisées dans les services aux personnes âgées. Par ailleurs, en Ontario, il y a maintenant une loi qui détermine qui a le droit d'utiliser le titre de «travailleur social». Je crois que cela aura pour effet de limiter le nombre de gens qui vont se définir comme travailleuses sociales. Par contre, les employeurs ne sont pas obligés, selon la loi, d'embaucher des gens avec des diplômes universitaires professionnels. Donc, malgré une limite au niveau de l'usage et du nombre de travailleuses sociales, ceci n'aura pas d'impact sur la demande. La profession du service social n'a pas encore réussi à avoir le contrôle sur la demande. La profession ne peut pas dire à un employeur d'embaucher une personne ayant une formation académique précise.

Ce qui n'aide pas, je crois, c'est que les écoles de service social n'ont pas réussi à définir clairement ce qui distingue un diplôme collégial, un diplôme universitaire de premier, deuxième ou troisième cycle, ni au niveau de la formation, ni à celui du travail. Par exemple, une personne avec une maitrise n'est pas nécessairement mieux préparée pour faire de la supervision qu'une personne avec un baccalauréat.

Reflets: Necroyez-vous pas que l'Association des travailleurs et travailleuses sociales d'Ontario (ATTSO) a un rôle à jouer ici?

Moscovitch: Même l'ATTSO n'a aucun contrôle sur la demande pour nos services. En Ontario, l'ATTSO et le Collège peuvent déterminer qui peut devenir membre, mais ils ne peuvent pas déterminer qui peut obtenir tel ou tel emploi dans les services sociaux. En général, je crois que la profession est en position d'attente. Actuellement, il n'y a pas une vision claire de l'avenir de notre profession.Aussi, s'il y a un défi que notre profession doit relever, c'est d'identifier et de définir notre vision de notre profession. Malheureusement, je ne crois pas qu'il y aura une solution rapide. 
Reflets: Vous soulevez des points très importants et il est clair qu'il n'y a pas de réponses faciles. Professeur Moscovitch, de la part de l'équipe de rédaction de la revue Reflets, nous vous remercions infiniment. Vous nous avez donné un défi à relever qui va nous tenir occupé pour quelques années. 